\title{
UPPER BOUND FOR PALINDROMIC AND FACTOR COMPLEXITY OF RICH WORDS
}

\author{
Josef RuKAVICKA*
}

\begin{abstract}
A finite word $w$ of length $n$ contains at most $n+1$ distinct palindromic factors. If the bound $n+1$ is attained, the word $w$ is called rich. An infinite word $w$ is called rich if every finite factor of $w$ is rich.

Let $w$ be a word (finite or infinite) over an alphabet with $q>1$ letters, let $\operatorname{Fac}_{w}(n)$ be the set of factors of length $n$ of the word $w$, and let $\operatorname{Pal}_{w}(n) \subseteq \operatorname{Fac}_{w}(n)$ be the set of palindromic factors of length $n$ of the word $w$.

We present several upper bounds for $\left|\operatorname{Fac}_{w}(n)\right|$ and $\left|\operatorname{Pal}_{w}(n)\right|$, where $w$ is a rich word. Let $\delta=$ $\frac{3}{2(\ln 3-\ln 2)}$. In particular we show that
\end{abstract}

$$
\left|\operatorname{Fac}_{w}(n)\right| \leq\left(4 q^{2} n\right)^{\delta \ln 2 n+2}
$$

In 2007, Baláži, Masáková, and Pelantová showed that

$$
\left|\operatorname{Pal}_{w}(n)\right|+\left|\operatorname{Pal}_{w}(n+1)\right| \leq\left|\operatorname{Fac}_{w}(n+1)\right|-\left|\operatorname{Fac}_{w}(n)\right|+2,
$$

where $w$ is an infinite word whose set of factors is closed under reversal. We prove this inequality for every finite word $v$ with $|v| \geq n+1$ and $\operatorname{Fac}_{v}(n+1)$ closed under reversal.

Mathematics Subject Classification. 68R15.

Received October 23, 2019. Accepted December 11, 2020.

\section{INTRODUCTION}

The field of combinatorics on words includes the study of palindromes and rich words. In recent years there have appeared several articles concerning this topic $[3,5,8,17]$. Recall that a palindrome is a word that is equal to its reversal, such as "noon" and "level". A word is called rich if it contains the maximal number of palindromic factors. It is known that a word of length $n$ can contain at most $n+1$ palindromic factors, including the empty word [8]. An infinite word $w$ is rich if every finite factor of $w$ is rich.

Rich words possess various properties; see, for instance $[4,7,9]$. We will use two of them. The first uses the notion of a complete return. Given a word $w$ and a factor $r$ of $w$, we call the factor $r$ a complete return to $u$

Keywords and phrases: Rich words, Palindromes, Palindromic complexity, Factor complexity.

Department of Mathematics, Faculty of Nuclear Sciences and Physical Engineering, Czech Technical University in Prague, 16636 Prague 6, Czechia.

* Corresponding author: josef.rukavicka@seznam.cz 
in $w$ if $r$ contains exactly two occurrences of $u$, one as a prefix and one as a suffix. A property of rich words is that all complete returns to any palindromic factor $u$ in $w$ are palindromes [9].

The second property of rich words that we use says that a factor $r$ of a rich word $w$ is uniquely determined by its longest palindromic prefix and its longest palindromic suffix [7]. Some generalizations of this property may be found in [12].

In the current article we present upper bounds for the palindromic and factor complexity of rich words. In other words, this means that we derive upper bounds for the number of palindromes and factors of given length in a rich word $w$. There are already some related results; see below.

We start with some results that hold for arbitrary (not only rich) words.

Let us define $\operatorname{Fac}_{w}(n)$ to be the set of factors of length $n$ of the word $w$, let $\operatorname{Pal}_{w}(n)$ be the set of palindromic factors of length $n$ of $w$, and let $\operatorname{Fac}_{w}=\bigcup_{j \geq 0} \operatorname{Fac}_{w}(j)$, where $w$ is a finite or infinite word. Let $w^{R}$ denote the reversal of $w=w_{1} w_{2} \ldots w_{n-1} w_{n}$, where $w_{i}$ are letters; formally $w^{R}=w_{n} w_{n-1} \ldots w_{2} w_{1}$. We say that a set $S$ of finite words is closed under reversal if $w \in S$ implies that $w^{R} \in S$.

It is clear that $\left|\operatorname{Pal}_{w}(n)\right| \leq\left|\operatorname{Fac}_{w}(n)\right|$. Some less obvious inequalities are known. One of the interesting inequalities is the following one [2,4]. If $w$ is an infinite word with $\mathrm{Fac}_{w}$ closed under reversal then

$$
\left|\operatorname{Pal}_{w}(n)\right|+|\operatorname{Pal}(w, n+1)| \leq\left|\operatorname{Fac}_{w}(n+1)\right|-\left|\operatorname{Fac}_{w}(n)\right|+2 .
$$

In [2] the authors proved the inequality (1.1) for uniformly recurrent words, but in the proof only "recurrent" is applied. It is known that if $\mathrm{Fac}_{w}$ is closed under reversal, then $w$ is recurrent ([6], Prop. 2.2). In Section 3 we generalize (1.1) for every finite word $v$ with $\operatorname{Fac}_{v}(n+1)$ closed under reversal, which allows us to improve our upper bound from Section 2 for the factor complexity of finite rich words. $\left.\left\lfloor\frac{n}{4}\right\rfloor\right) \mid$.

In [1], another inequality has been proven for infinite non-ultimately periodic words: $\left|\operatorname{Pal}_{w}(n)\right|<\frac{16}{n} \mid \operatorname{Fac}_{w}(n+$

In [14], the authors show that a random word of length $n$ contains, on expectation, $\Theta(\sqrt{n})$ distinct palindromic factors.

Now, let us focus on rich words.

Let $\Pi(n)$ denote the number of rich words of length $n$. If $w$ is a rich word then obviously $\left|\operatorname{Fac}_{w}(n)\right| \leq \Pi(n)$. Hence the number of rich words forms the upper bound for the palindromic and factor complexity of rich words. The number of rich words was investigated in [19], where the author gives a recursive lower bound on the number of rich words of length $n$, and an upper bound on the number of binary rich words. Better results can be found in [10]. The authors of [10] construct for each $n$ a large set of rich words of length $n$. Their construction gives, currently, the best lower bound on the number of binary rich words, namely

$$
\Pi(n) \geq \frac{C^{\sqrt{n}}}{p(n)},
$$

where $p(n)$ is a polynomial and the constant $C \approx 37$.

Every factor of a rich word is also rich [9]. In other words, the language of rich words is factorial. In particular, this means that $\Pi(n) \Pi(m) \geq \Pi(n+m)$ for all $m, n \in \mathbb{N}$. Therefore, Fekete's lemma implies the existence of the limit of $\sqrt[n]{\Pi(n)}$, and moreover [10]:

$$
\lim _{n \rightarrow \infty} \sqrt[n]{\Pi(n)}=\inf \{\sqrt[n]{\Pi(n)}: n \in \mathbb{N}\}
$$

For a fixed $n_{0}$, one can find the number of all rich words of length $n_{0}$ and obtain an upper bound on the limit. Using a computer Rubinchik counted $\Pi(n)$ for $n \leq 60$; see the sequence https://oeis.org/A216264. As $\sqrt[60]{\Pi(60)}<1.605$, he obtained an upper bound for the binary alphabet: $\Pi(n)<c 1.605^{n}$ for some constant $c$ $[10]$. 
In [15], the author shows that $\Pi(n)$ has a subexponential growth on every finite alphabet. Formally $\lim _{n \rightarrow \infty} \sqrt[n]{\Pi(n)}=1$. This result is an argument in favor of a conjecture formulated in [10] saying that for some infinitely growing function $g(n)$ the following holds for a binary alphabet:

$$
\Pi(n)=\mathcal{O}\left(\frac{n}{g(n)}\right)^{\sqrt{n}}
$$

As already mentioned, we construct upper bounds for palindromic and factor complexity of rich words. The proof uses the following idea. Let $u$ be a palindromic factor of a rich word $w$ on the alphabet $A$, such that $a u b$ is factor of $w$, where $a, b \in A$ and $a \neq b$. Let $\operatorname{lpp}(w)$ and $\operatorname{lps}(w)$ denote the longest palindromic prefix and suffix of $w$ respectively. Then $\operatorname{lpp}(a u b)$ and $\operatorname{lps}(a u b)$ uniquely determine the factor $a u b$ in $w$ [7]. Let $\operatorname{lpps}(w)$ denote the longest proper palindromic suffix of $w$. We show that $a, b$ and $\operatorname{lpps}(u)$ also uniquely determine $a u b$. In addition, we observe that either $|\operatorname{lpps}(u)| \leq \frac{1}{2}|u|$ or $u$ contains a palindromic factor $\bar{u}$ that uniquely determines $u$ and $|\bar{u}| \leq \frac{1}{2}|u|$. We obtain a "short" palindrome and letters $a, b$ that uniquely determine the "long" palindrome $u$ in the case when $a u b$ is a factor of $w$. In these "short" palindromes there are again other "shorter" palindromes, and so on. As a consequence we present an upper bound for the number of factors of the form $a u b$ with $|a u b|=n$. The property of rich words that all complete returns to any palindromic factor $u$ in $w$ are palindromes [9] allows us to prove that if $w$ contains the factors $x u x$ and $y u y$, where $x, y \in A$ and $x \neq y$, then $w$ must contain a factor of the form $a u b$, where $a, b \in A$ and $a \neq b$. This property demonstrates the relation between the factors $a u b$ and palindromic factors $x u x$. Due to this we derive an upper bound for the palindromic complexity of rich words. With the upper bound for palindromic complexity, the property that each factor is uniquely determined by its longest palindromic prefix and suffix [7], and the inequality (1.1) we obtain several upper bounds on palindromic and factor complexity. The main result of the current article is the following theorem.

Theorem 1.1. If $\delta=\frac{3}{2(\ln 3-\ln 2)}, w$ is a finite or infinite rich word over an alphabet with $q>1$ letters, and $n$ is a positive integer then

$$
\left|\operatorname{Fac}_{w}(n)\right| \leq\left(4 q^{2} n\right)^{\delta \ln 2 n+2}
$$

The main result is a quasi-polynomial upper bound for factor complexity of rich words. This is much less than the lower bound on the number of rich words; recall (1.2). Thus an infinite rich word can contain only a small share of all finite rich words. This contrasts with power-free languages, where an infinite word can contain all extendable finite words with the same power-freeness restriction [13, 16, 18].

\section{PALINDROMIC AND FACTOR COMPLEXITY OF RICH WORDS}

Consider an alphabet $A$ with $q$ letters, where $q>1$. Let $A^{+}=\bigcup_{j>0} A^{j}$ denote the set of all nonempty words over $A$, where $A^{j}$ is the set of words of length $j$.

Let $\epsilon$ denote the empty word, let $A^{*}=A^{+} \cup\{\epsilon\}$, and let

$$
A^{\infty}=\left\{w_{1} w_{2} w_{3} \ldots \mid w_{i} \in A \text { and } i>0\right\}
$$

be the set of infinite words.

Let $R_{n} \subseteq A^{n}$ be the set of rich words of length $n \geq 0$. Let $R^{+}=\bigcup_{j>0} R_{j}$ and $R^{*}=R^{+} \cup\{\epsilon\}$. In addition, we define $R^{\infty} \subseteq A^{\infty}$ to be the set of infinite rich words. Let $R=R^{+} \cup R^{\infty}$.

Let $\operatorname{lps}(w)$ and $\operatorname{lpp}(w)$ be the longest palindromic suffix and the longest palindromic prefix of a word $w \in A^{*}$ respectively. Additionally, we introduce $\operatorname{lpps}(w)$ to be the longest proper palindromic suffix and $\operatorname{lppp}(w)$ to be the longest proper palindromic prefix, where $|w|>1$; proper means that $\operatorname{lpps}(w) \neq w$ and $\operatorname{lppp}(w) \neq w$. For a word $w$ with $|w| \leq 1$ we define $\operatorname{lppp}(w)=\operatorname{lpps}(w)=\epsilon$. 
Let $w=w_{1} w_{2} \ldots w_{n}$ be a word, where $w_{i} \in A$. We define $w[i]=w_{i}$ and $w[i, j]=w_{i} w_{i+1} \ldots w_{j}$, where $0<i \leq j \leq n$.

Moreover we define the following notation:

$-\mathrm{P}_{n} \subset A^{n}$ : the set of palindromes of length $n \geq 0$.

- $\mathrm{P}^{+}=\bigcup_{j>0} \mathrm{P}_{j}$ (the set of all nonempty palindromes).

- $\mathrm{Fac}_{w}$ : the set of factors of the word $w \in A^{*} \cup A^{\infty}$.

$-\operatorname{Fac}_{w}(n)=\left\{u \mid u \in \operatorname{Fac}_{w}\right.$ and $\left.|u|=n\right\}$ (the set of factors of length $n$ ).

- $\mathrm{Pal}_{w}=\left(\mathrm{P}^{+} \cup\{\epsilon\}\right) \cap \mathrm{Fac}_{w}$ (the set of palindromic factors).

$-\operatorname{Pal}_{w}(n)=\operatorname{Fac}_{w}(n) \cap \mathrm{P}_{n}$ (the set of palindromic factors of length $n$ ).

Definition 2.1. Let $\operatorname{trim}(w)=w[2,|w|-1]$, where $w \in A^{*}$ and $|w|>2$. For $|w| \leq 2$ we define $\operatorname{trim}(w)=\epsilon$. If $S$ is a set of words, then

$$
\operatorname{trim}(S)=\{\operatorname{trim}(v) \mid v \in S\}
$$

Remark 2.2. The function $\operatorname{trim}(w)$ removes the first and last letter from $w$.

Example 2.3. Suppose that $A=\{0,1,2,3,4,5\}$.

$-\operatorname{trim}(01123501)=112350$.

$-\operatorname{trim}(\{12213,112,2,344\})=\{221,1, \epsilon, 4\}$.

We will deal a lot with the words of the form $a u b$, where $u$ is a palindrome and $a, b$ are distinct letters. Hence we introduce some more notation for them.

Definition 2.4. Given $w \in R$ and $n>2$, let

$$
\begin{array}{r}
\mathrm{Sw}_{w}(n)=\left\{a u b \mid a u b \in \operatorname{Fac}_{w}(n) \text { and } u \in \operatorname{Pal}_{w}(n-2)\right. \\
\text { and } a, b \in A \text { and } a \neq b\} .
\end{array}
$$

If $n \leq 2$ then we define $\mathrm{Sw}_{w}(0)=\mathrm{Sw}_{w}(1)=\mathrm{Sw}_{w}(2)=\emptyset$.

Let $\overline{\mathrm{Sw}}_{w}(n)=\bigcup_{a u b \in \mathrm{Sw}_{w}(n)}\{(u, a),(u, b)\}$, where $a, b \in A$. Let $a u b \in \mathrm{Sw}_{w}(n)$, where $a, b \in A$. We call the word $a u b$ a $u$-switch of $w$. Alternatively we say that $w$ contains a $u$-switch.

Remark 2.5. Note that a pair $(u, a) \in \overline{\mathrm{Sw}}_{w}(n)$ if and only if there exists $b \in A$ such that $a u b \in \mathrm{Sw}_{w}(n)$ or bua $\in \mathrm{Sw}_{w}(n)$.

Example 2.6. If $A=\{0,1,2,3,4,5,6\}$ and

$$
w=5112211311001131133114111146
$$

then:

$-\mathrm{Sw}_{w}(8)=\{51122113,31133114,14111146\}$.

$-\operatorname{trim}\left(\mathrm{Sw}_{w}(8)\right)=\{112211,113311,411114\}$.

$-\mathrm{Sw}_{w}(8)=\{(112211,3),(112211,5),(113311,3),(113311,4)$, $(411114,1),(411114,6)\}$.

- $w$ does not contain 110011-switch. Formally $110011 \notin \operatorname{trim}\left(\operatorname{Sw}_{w}(8)\right)$.

Remark 2.7. The idea of a $u$-switch is inspired by the next lemma. If a rich word $w$ contains palindromes $a u a, b u b$, where $a, b \in A, a \neq b$, and $|a u a|=|b u b|=n$, then $w$ contains a $u$-switch of length $n$. The $u$-switch "switches" from $a$ to $b$. Note that $a u a, b u b \in \mathrm{Fac}_{w}$ does not imply that $a u b \in \mathrm{Fac}_{w}$ or $b u a \in \mathrm{Fac}_{w}$. It may be, for example, that $a u c, c u b \in \operatorname{Fac}_{w}$. Nonetheless $(u, a),(u, b) \in \widehat{\mathrm{Sw}}_{w}(n)$. 
Lemma 2.8. Suppose $w \in R$ and suppose $u \in \operatorname{Pal}_{w}(n-2)$, where $n>2$. If $a, b_{1}, b_{2} \in A,\left|\left\{a, b_{1}, b_{2}\right\}\right|>1$, and $a u a, b_{1} u b_{2} \in \operatorname{Fac}_{w}(n)$ then $(u, a) \in \overline{\operatorname{Sw}}_{w}(n)$.

Remark 2.9. The condition $\left|\left\{a, b_{1}, b_{2}\right\}\right|>1$ in Lemma 2.8 means that at least one letter is different from the others.

Proof. Let $r$ be a factor of $w$ such that aua is unioccurrent in $r$ and $\operatorname{trim}(r)$ is a complete return to $u$ in $w$. Since $a u a$ and $b_{1} u b_{2}$ are factors of $w$, it is obvious that such $r$ exists. Clearly there are $x_{1}, x_{2}, y_{1}, y_{2} \in A$ such that $x_{1} u x_{2}$ is a prefix of $r$ and $y_{1} u y_{2}$ is a suffix of $r$. The complete return $\operatorname{trim}(r)$ to $u$ is a palindrome [9]. Hence $x_{2}=y_{1}$. Since aua is unioccurrent in $r$, it follows that $x_{2}=y_{1}=a, x_{1} \neq y_{2}$, and $a \in\left\{x_{1}, y_{2}\right\}$. In consequence we have that $(u, a) \in \overline{\mathrm{Sw}}_{w}(n)$.

To clarify the previous proof, let us consider the following two examples. For both examples suppose that $A=\{1,2,3,4,5,6\}$.

Example 2.10. Let $w=321234321252126$. Let $a u a=32123$ and $b_{1} u b_{2}=52126$. Then $r=32123432125$ and $\operatorname{trim}(r)=212343212$ is a complete return to 212 . Therefore $(212,3) \in \overline{\mathrm{Sw}}_{w}(5)$. Note that $b_{1} u b_{2}$ is not a factor of $r$.

Example 2.11. Let $w=321234321252$. Let $a u a=32123$ and $x u y=b_{1} u b_{2}=32125$. Then $r=32123432125$ and $\operatorname{trim}(r)=212343212$ is a complete return to 212 . Therefore $(212,3) \in \mathrm{Sw}_{w}(5)$. Note that $b_{1} u b_{2}$ is a factor of $r$.

We show that the number of palindromic factors and the number of $u$-switches are related.

Proposition 2.12. If $w \in R$ and $n>2$ then

$$
2\left|\mathrm{Sw}_{w}(n)\right|+\left|\operatorname{Pal}_{w}(n-2)\right| \geq\left|\operatorname{Pal}_{w}(n)\right| .
$$

Proof. Let $\omega(w, n)=\left\{a u a \mid(u, a) \in \overline{\mathrm{Sw}}_{w}(n)\right\}$. Less formally said, $\omega(w, n)$ is a set of palindromes of length $n$ such that if $w$ contains a $u$-switch $a u b$ then $a u a, b u b \in \omega(w, n)$. Obviously we have that

$$
|\omega(w, n)| \leq 2\left|\mathrm{Sw}_{w}(n)\right| .
$$

Let

$$
\tilde{\mathrm{Pal}_{w}}(n)=\left\{v \mid v \in \operatorname{Pal}_{w}(n) \text { and } \operatorname{trim}(v) \in \operatorname{trim}\left(\operatorname{Sw}_{w}(n)\right)\right\}
$$

and

$$
\operatorname{Pal}_{w}(n)=\left\{v \mid v \in \operatorname{Pal}_{w}(n) \text { and } \operatorname{trim}(v) \notin \operatorname{trim}\left(\mathrm{Sw}_{w}(n)\right)\right\} .
$$

Obviously $\operatorname{Pal}_{w}(n)=\tilde{\operatorname{Pal}_{w}}(n) \cup \dot{\operatorname{Pal}_{w}}(n)$ and $\tilde{\operatorname{Pal}_{w}}(n) \cap \dot{\operatorname{Pal}_{w}}(n)=\emptyset$. It follows that

$$
\left|\tilde{P a l}_{w}(n)\right|+\left|\dot{\operatorname{Pal}_{w}}(n)\right|=\left|\operatorname{Pal}_{w}(n)\right| \text {. }
$$

Suppose $v \in \operatorname{Pal}_{w}(n)$ and let $u=\operatorname{trim}(v)$.

- If $v \in \tilde{\operatorname{Pal}}_{w}(n)$ then $w$ contains a $u$-switch. From Lemma 2.8 it follows that $v \in \omega(w, n)$; this and $(2.1)$ imply that

$$
\left|\tilde{\mathrm{Pal}_{w}}(n)\right| \leq|\omega(w, n)| \leq 2\left|\mathrm{Sw}_{w}(n)\right| .
$$


- If $v \notin \tilde{\mathrm{Pal}_{w}}(n)$ then $w$ does not contain a $u$-switch. We have that $u \in \operatorname{Pal}_{w}(n-2) \backslash \operatorname{trim}\left(\mathrm{Sw}_{w}(n)\right)$. Obviously if $t \in \mathrm{Pal}_{w}(n-2) \backslash \operatorname{trim}\left(\mathrm{Sw}_{w}(n)\right), a, b \in A$, and $w$ has palindromic factors ata and $b t b$, then $a=b$ since $w$ does not contain a $t$-switch. It follows that

$$
\left|\dot{\operatorname{Pal}_{w}}(n)\right| \leq\left|\operatorname{Pal}_{w}(n-2)\right|
$$

The proposition follows from (2.2), (2.3), and (2.4).

To clarify the previous proof, let us consider the following example.

Example 2.13. If $A=\{0,1,2,3,4,5,6,7,8\}$ and

$$
w=2110112333211011454110116110116778776
$$

then

$-\mathrm{Sw}_{w}(7)=\{2110114,4110116\}$,

- $\mathrm{Pal}_{w}(7)=\{1233321,2110112,1145411,6110116,6778776\}$,

$-\tilde{\mathrm{Pal}}_{w}(7)=\{2110112,6110116\}$,

$-\operatorname{Pal}_{w}(7)=\{1233321,1145411,6778776\}$,

$-\mathrm{Pal}_{w}(5)=\{23332,11011,14541,77877\}$,

$-2\left|\mathrm{Sw}_{w}(7)\right|+\left|\mathrm{Pal}_{w}(5)\right| \geq\left|\mathrm{Pal}_{w}(7)\right|$, and

$-4+4>5$.

In the next proposition we show that if $a, b$ are different letters and $a u b$ is a switch of a rich word $w$ then the longest proper palindromic suffix $r$ of $u$ and the letters $a, b$ uniquely determine the palindromic factor $u \in \mathrm{Pal}_{w}$.

Proposition 2.14. If $w \in R, u, v \in \operatorname{Pal}_{w}, \operatorname{lpps}(u)=\operatorname{lpps}(v), a, b \in A, a \neq b$, and $a u b, a v b \in \mathrm{Fac}_{w}$ then $u=v$.

Proof. It is known that if $r, t$ are two factors of a rich word $w$ and $\operatorname{lps}(r)=\operatorname{lps}(t)$ and $\operatorname{lpp}(r)=\operatorname{lpp}(t)$, then $r=t[7]$. We will identify a $u$-switch by the longest proper palindromic suffix of $u$ and two distinct letters $a, b$ instead of by the functions lps and lpp.

Given a $u$-switch $a u b$ where $a \neq b, a, b \in A$, we know that $\operatorname{lps}(a u b)$ and $\operatorname{lpp}(a u b)$ uniquely determine the factor $a u b$ in $w$. We will prove that for given $a, b \in A, a \neq b, n \geq 0$, and a palindrome $r$ there is at most one palindrome $u \in \mathrm{Pal}_{w}$ such that $\operatorname{lpps}(u)=r$ and $a u b \in \mathrm{Sw}_{w}(|a u b|)$.

Suppose, to get a contradiction, that there are $u, v \in \mathrm{Pal}_{w}, u \neq v, a, b \in A, a \neq b$ such that $\operatorname{lps}(a u b)=$ $b p b, \operatorname{lps}(a v b)=b s b, \operatorname{lpp}(a u b)=a x a, \operatorname{lpp}(a v b)=a y a, \operatorname{lpps}(u)=\operatorname{lpps}(v)=r$, and $a u b, a v b \in \bigcup_{j>0} \mathrm{Sw}_{w}(j)$. This implies that $p, s, x, y$ are prefixes of $r$. Thus if $x \neq y$, then $|x| \neq|y|$. Without loss of generality, let $|x|<|y|$. Since $y$ is a prefix of $r$, either ya is a prefix of $r$ or $r=y$. Consequently aya is a prefix of both $a u b$ and $a v b$, and this contradicts the assumption that $\operatorname{lpp}(a u b)=a x a$; aya is a prefix of $a u b$ and $|a y a|>|a x a|$. Analogously if $p \neq s$. It follows that $x=y$ and $p=s$. Therefore $\operatorname{lpp}(a u b)=\operatorname{lpp}(a v b)$ and $\operatorname{lps}(a u b)=\operatorname{lps}(a v b)$, which would imply that $u=v$, which is a contradiction.

Hence we conclude that $a, b \in A, a \neq b$, and a palindrome $r$ determine at most one palindrome $u \in \mathrm{Pal}_{w}$ such that $\operatorname{lpps}(u)=r$ and $u \in \operatorname{trim}\left(\operatorname{Sw}_{w}(|u|+2)\right)$.

In the following we derive an upper bound for the number of $u$-switches. We need one more definition to be able to partition the set $\mathrm{Sw}_{w}(n)$ into subsets based on the longest proper palindromic suffix.

Definition 2.15. Given $w \in R, r \in R^{+}$and $n \geq 0$, let

$$
\Upsilon_{w}(n, r)=\left\{u \mid u \in \mathrm{Sw}_{w}(n) \text { and } \operatorname{lpps}(\operatorname{trim}(u))=r\right\}
$$


Remark 2.16. The set $\Upsilon_{w}(n, r)$ contains switches $a v b$ of length $n$ of the word $w$ such that the longest proper palindromic suffix of $v$ equals to $r$, where $a, b$ are letters. Obviously $\bigcup_{r \in \operatorname{Pal}_{w}} \Upsilon_{w}(n, r)=\operatorname{Sw}_{w}(n)$ and $\Upsilon_{w}(n, r) \cap$ $\Upsilon_{w}(n, \bar{r})=\emptyset$ if $r \neq \bar{r}$.

A simple corollary of the previous proposition is that the size of the set $\Upsilon_{w}(n, r)$ is limited by the constant $q(q-1)$. Recall that $q$ is the size of the alphabet $A$.

Corollary 2.17. If $w, r \in R$ and $n \geq 0$ then $\left|\Upsilon_{w}(n, r)\right| \leq q(q-1)$.

Proof. From Proposition 2.14 it follows that

$$
\left|\Upsilon_{w}(n, r)\right| \leq \mid\{(a, b) \mid a, b \in A \text { and } a \neq b\} \mid=q(q-1) .
$$

In other words, $\left|\Upsilon_{w}(n, r)\right|$ is equal or smaller that the number of pairs of distinct letters $(a, b)$.

We define $\bar{\Gamma}_{w}(n)=\max \left\{\left|\mathrm{Sw}_{w}(i)\right| \mid 0 \leq i \leq n\right\}$, where $w \in R$ and $n \geq 0$. Furthermore we define $\Gamma_{w}(n)=$ $\max \left\{q, \bar{\Gamma}_{w}(n)\right\}$.

Remark 2.18. We defined $\Gamma_{w}(n)$ as the maximum from the set of sizes of $\mathrm{Sw}_{w}(i)$, where $0 \leq i \leq n$. In addition, we defined that $\Gamma_{w}(n) \geq q$. This is just for practical reason to make the formulas easier; since we look for upper bounds, this simplification is justified. The function $\Gamma_{w}(n)$ will allow us to present another relation between the number of palindromic factors of length $n$ and the number of $u$-switches without using $\operatorname{Pal}_{w}(n-2)$.

Lemma 2.19. If $w \in R$ and $n>0$ then

$$
n \Gamma_{w}(n) \geq\left|\operatorname{Pal}_{w}(n)\right|
$$

Proof. We define two functions $\bar{\phi}$ and $\phi$ as follows. If $n$ is even then $\bar{\phi}(n)=2$, otherwise $\bar{\phi}(n)=1$. Let $\phi(n)=$ $\{2+\bar{\phi}(n), 4+\bar{\phi}(n), \ldots, n\}$. For example $\phi(8)=\{4,6,8\}$ and $\phi(9)=\{3,5,7,9\}$.

Proposition 2.12 states that

$$
2\left|\mathrm{Sw}_{w}(n)\right|+\left|\operatorname{Pal}_{w}(n-2)\right| \geq\left|\operatorname{Pal}_{w}(n)\right| .
$$

It follows that

$$
2\left|\mathrm{Sw}_{w}(n-2)\right|+\left|\operatorname{Pal}_{w}(n-4)\right| \geq\left|\operatorname{Pal}_{w}(n-2)\right| .
$$

From (2.5) and (2.6):

$$
2\left|\mathrm{Sw}_{w}(n)\right|+2\left|\mathrm{Sw}_{w}(n-2)\right|+\operatorname{Pal}_{w}(n-4)|\geq| \operatorname{Pal}_{w}(n) \mid .
$$

In general (2.5) implies that

$$
2\left|\mathrm{Sw}_{w}(n-i)\right|+\left|\operatorname{Pal}_{w}(n-2 i)\right| \geq\left|\operatorname{Pal}_{w}(n-i)\right| .
$$

Then by iterative applying of (2.8) to (2.7) we obtain that

$$
\sum_{j \in \phi(n)} 2\left|\mathrm{Sw}_{w}(j)\right|+\left|\operatorname{Pal}_{w}(\bar{\phi}(n))\right| \geq\left|\operatorname{Pal}_{w}(n)\right|
$$

We have that $\left|\operatorname{Pal}_{w}(\bar{\phi}(n))\right| \leq q$; just consider that $\left|\operatorname{Pal}_{w}(\bar{\phi}(n))\right|$ is the number of palindromes of length 1 or 2. Recall that $\Gamma_{w}(n) \geq\left|\mathrm{Sw}_{w}(j)\right|$ for $2<j<n$ and realize that $|\phi(n)| \leq \frac{n-1}{2}$. It follows from (2.9) that 
$(n-1) \Gamma_{w}(n)+q \geq\left|\operatorname{Pal}_{w}(n)\right|$. It is easy to see that $n \Gamma_{w}(n) \geq(n-1) \Gamma_{w}(n)+q$ for $n>0$, since $\Gamma_{w}(n) \geq q$. This completes the proof.

We will need to cope with the longest proper palindromic suffixes that are "too long". We show that if the longest proper palindromic suffix $\operatorname{lpps}(v)$ is longer the half of the length of $v$, then $v$ contains a "short" palindromic factor, that uniquely determines $v$. We will use the two following lemmas from [11]:

Lemma 2.20. (see [11], Lem. 1) Suppose $p$ is a period of a nonempty palindrome $w$; then there are palindromes $a$ and $b$ such that $|a b|=p, b \neq \epsilon$, and $w=(a b)^{j} a$ for some non-negative integer $j$.

Lemma 2.21. (see [11], Lem. 2) Suppose $w$ is a palindrome and $u$ is its proper suffix-palindrome or prefixpalindrome; then the number $|w|-|u|$ is a period of $w$.

Let $u, v \in \mathrm{P}^{+}$such that $u$ is a suffix of $v$ and $|u|<|v|$. Lemma 2.21 implies that $v$ is periodic with period $p=|v|-|u|$. Lemma 2.20 implies that there are palindromes $a, b$ such that $b$ is nonempty and $p=|a b|$ and $v=(a b)^{j} a$ for some non-negative integer $j$. We define $\bar{\rho}(u, v)=(a, b)$ and $\rho(u, v)=a b a \in \mathrm{P}^{+}$.

The next lemma is an obvious consequence of Lemma 2.20 and Lemma 2.21. It says that $v$ is uniquely determined by the palindrome $\rho(u, v)$ and by the lengths of $u$ and $v$.

Lemma 2.22. If $u_{1}, u_{2}, v_{1}, v_{2} \in \mathrm{P}^{+},\left|v_{1}\right|=\left|v_{2}\right|,\left|u_{1}\right|=\left|u_{2}\right|,\left|u_{1}\right|<\left|v_{1}\right|, u_{1}$ is a suffix of $v_{1}, u_{2}$ is a suffix of $v_{2}$, and $\rho\left(u_{1}, v_{1}\right)=\rho\left(u_{2}, v_{2}\right)$ then $v_{1}=v_{2}$.

Proof. Let $\bar{\rho}\left(u_{1}, v_{1}\right)=\left(a_{1}, b_{1}\right)$ and let $\rho\left(u_{2}, v_{2}\right)=\left(a_{2}, b_{2}\right)$. Let $p=\left|v_{1}\right|-\left|u_{1}\right|=\left|v_{2}\right|-\left|u_{2}\right|$. Since $\rho\left(u_{1}, v_{1}\right)=$ $\rho\left(u_{2}, v_{2}\right)$, from Lemma 2.20 and Lemma 2.21 we have that $p=\left|a_{1} b_{1}\right|=\left|a_{2} b_{2}\right|$. Also it follows that $a_{1} b_{1}=a_{2} b_{2}$ and $a_{1} b_{1} a_{1}=a_{2} b_{2} a_{2}$. In consequence we get that $a_{1}=a_{2}$ and $b_{1}=b_{2}$. This ends the proof.

In the next lemma we consider a palindromic suffix $u$ of a palindrome $v$, which is longer than the half of $v$. For this case we show an upper bound for the length of the palindrome $\rho(u, v)$.

Lemma 2.23. If $u, v \in \mathrm{P}^{+}, u$ is a suffix of $v$, and $\frac{1}{2}|v| \leq|u|<|v|$ then

$$
\rho(u, v) \leq\left\lceil\frac{2}{3}|v|\right\rceil
$$

Proof. Let $(a, b)=\bar{\rho}(u, v)$. It is easy to verify that $\frac{1}{2}|v| \leq|u|<|v|$ implies that $j \geq 2$, where $v=(a b)^{j} a$.

Let $c$ be a positive real constant such that $|a b a|=c\left|(a b)^{j} a\right|$. For given $a, b$ it is clear that $c$ decreases as $j$ increases. Since $j>1$ it follows that $c$ is maximal for $j=2$. Thus $c \leq \frac{|a b a|}{|a b a b a|}=\frac{2|a|+|b|}{3|a|+2|b|}$. The lemma follows.

We derive an upper bound for the number of $u$-switches.

Proposition 2.24. If $w \in R$ and $n>2$ then

$$
\Gamma_{w}(n) \leq 2 q^{2}\left(\frac{2 n}{3}\right)^{3} \Gamma_{w}\left(\left\lfloor\frac{2 n}{3}\right\rfloor\right) .
$$

Proof. We partition the set $\mathrm{Sw}_{w}(n)$ into sets $\Delta_{\rho}(w, n), \Delta_{\text {lpps }}(w, n)$ as follows. Let $a v b \in \mathrm{Sw}_{w}(n)$ be a $v$-switch, where $a, b \in A$. If $\frac{1}{2}|v| \leq|\operatorname{lpps}(v)|$ then $a v b \in \Delta_{\rho}(w, n)$ otherwise $a v b \in \Delta_{\text {lpps }}(w, n)$. Obviously $\Delta_{\rho}(w, n) \cap$ $\Delta_{\text {lpps }}(w, n)=\emptyset$ and

$$
\operatorname{Sw}_{w}(n)=\Delta_{\rho}(w, n) \cup \Delta_{\text {lpps }}(w, n) .
$$

Let us investigate the sizes of $\Delta_{\rho}(w, n)$ and $\Delta_{\text {lpps }}(w, n)$.

- If $a v b \in \Delta_{\rho}(w, n)$ then let $u=\operatorname{lpps}(v)$. We have that $\rho(u, v),|u|$, and $|v|$ uniquely determine the palindrome $v$; see Lemma 2.22. In addition, $|\rho(u, v)| \leq\left\lceil\frac{2|v|}{3}\right\rceil$; see Lemma 2.23. Realize that $|v|=n-2$; then the 
number of all palindromic factors of $w$ of length $\leq\left\lceil\frac{2(n-2)}{3}\right\rceil$ multiplied by $\left\lceil\frac{n-2}{2}\right\rceil$ (the number of different values of $|u|)$ must be bigger or equal to the size of $\operatorname{trim}\left(\Delta_{\rho}(w, n)\right)$. Realize that the set $\operatorname{trim}\left(\Delta_{\rho}(w, n)\right)$ contains palindromes of length $n-2$. Since $\left\lceil\frac{2(n-2)}{3}\right\rceil \leq\left\lfloor\frac{2 n}{3}\right\rfloor$ we have that

$$
\left|\operatorname{trim}\left(\Delta_{\rho}(w, n)\right)\right| \leq\left\lceil\frac{n-2}{2}\right\rceil \sum_{j=1}^{\left\lfloor\frac{2 n}{3}\right\rfloor}\left|\operatorname{Pal}_{w}(j)\right| .
$$

Since $a, b$ are distinct letters it follows that

$$
\left.\mid \Delta_{\rho}(w, n)\right)|\leq q(q-1)| \operatorname{trim}\left(\Delta_{\rho}(w, n)\right) \mid .
$$

- If $a v b \in \Delta_{\text {lpps }}(w, n)$ then $|\operatorname{lpps}(v)|<\frac{1}{2}|v|=\frac{n-2}{2}$. Obviously we have that

$$
\Delta_{\text {lpps }}(w, n)=\bigcup_{r \in S} \Upsilon_{w}(n, r) \text {, where } S=\left\{r \mid r \in \operatorname{Pal}_{w} \text { and }|r|<\frac{n-2}{2}\right\} .
$$

Since $\left\lceil\frac{n-2}{2}\right\rceil \leq\left\lfloor\frac{n}{2}\right\rfloor$ we have from Corollary 2.17 and (2.13) that

$$
\left|\Delta_{\text {lpps }}\right| \leq q(q-1) \sum_{j=1}^{\left\lfloor\frac{n}{2}\right\rfloor}\left|\operatorname{Pal}_{w}(j)\right| .
$$

It follows from (2.10), (2.11), (2.12), and (2.14) that

$$
\left|\mathrm{Sw}_{w}(n)\right| \leq 2 q(q-1)\left\lceil\frac{n-2}{2}\right\rceil \sum_{j=1}^{\left\lfloor\frac{2 n}{3}\right\rfloor}\left|\operatorname{Pal}_{w}(j)\right| .
$$

From Lemma 2.19 we know that $\left|\operatorname{Pal}_{w}(j)\right| \leq j \Gamma_{w}(j)$. Therefore we have that

$$
\sum_{j=1}^{\left\lfloor\frac{2 n}{3}\right\rfloor}\left|\operatorname{Pal}_{w}(j)\right| \leq \sum_{j=1}^{\left\lfloor\frac{2 n}{3}\right\rfloor} j \Gamma_{w}(j) \leq \frac{2 n}{3} \frac{2 n}{3} \Gamma_{w}\left(\left\lfloor\frac{2 n}{3}\right\rfloor\right) .
$$

To simplify the formulas, we apply that $q(q-1)<q^{2}$ and that $\left\lceil\frac{n-2}{2}\right\rceil \leq \frac{2 n}{3}$. From (2.15) and (2.16):

$$
\left|\mathrm{Sw}_{w}(n)\right| \leq 2 q^{2}\left(\frac{2 n}{3}\right)^{3} \Gamma_{w}\left(\left\lfloor\frac{2 n}{3}\right\rfloor\right)
$$

From Definition of $\Gamma_{w}(n)$ and (2.17) we get that

$$
\Gamma_{w}(n)=\max \left\{q, \max \left\{\left|\mathrm{Sw}_{w}(j)\right| \mid 0 \leq j \leq n\right\}\right\} \leq 2 q^{2}\left(\frac{2 n}{3}\right)^{3} \Gamma_{w}\left(\left\lfloor\frac{2 n}{3}\right\rfloor\right) .
$$

This ends the proof.

We will need the following lemma in the proof of Corollary 2.26.

Lemma 2.25. If $\beta>1$ is a real constant then $\prod_{j \geq 1}^{k} \frac{n}{\beta^{j}} \leq n^{\frac{\ln n}{2 \ln \beta}}$, where $k=\left\lfloor\frac{\ln n}{\ln \beta}\right\rfloor$. 
Proof.

$$
\prod_{j \geq 1}^{k} \frac{n}{\beta^{j}}=\frac{n}{\beta} \frac{n}{\beta^{2}} \frac{n}{\beta^{3}} \ldots \frac{n}{\beta^{k-1}} \frac{n}{\beta^{k}} \leq \frac{n^{k}}{\prod_{j=1}^{k} \beta^{j}} .
$$

We have that

$$
\prod_{j=1}^{k} \beta^{j}=\beta \beta^{2} \beta^{3} \ldots \beta^{k-1} \beta^{k}=\beta^{\sum_{j=1}^{k} j}=\beta^{\frac{k(k+1)}{2}} .
$$

Then from (2.18) and (2.19): $\prod_{j \geq 1}^{k} \frac{n}{\beta^{j}} \leq \frac{n^{k}}{\beta^{\frac{k(k+1)}{2}}}=\left(\frac{n}{\beta^{\frac{(k+1)}{2}}}\right)^{k}$.

Since $\beta^{k+1} \geq n$ :

$\left(\frac{n}{\beta^{\frac{(k+1)}{2}}}\right)^{k} \leq\left(\frac{n}{n^{\frac{1}{2}}}\right)^{k}=\left(n^{\frac{1}{2}}\right)^{k} \leq n^{\frac{\ln n}{2 \ln \beta}}$. This completes the proof.

In order to simplify the notation let $\alpha=\frac{3}{2}$ and let $\delta=\frac{3}{2 \ln \alpha}=\frac{3}{2(\ln 3-\ln 2)}$. Based on Proposition 2.24 we will derive a non-recurrent upper bound for $\Gamma_{w}(n)$.

Corollary 2.26. If $w \in R$, and $n>2$ then

$$
\Gamma_{w}(n) \leq q\left(2 q^{2} n\right)^{\delta \ln n}
$$

Proof. Proposition 2.24 states that

$$
\Gamma_{w}(n) \leq 2 q^{2}\left(\frac{n}{\alpha}\right)^{3} \Gamma_{w}\left(\left\lfloor\frac{n}{\alpha}\right\rfloor\right) .
$$

Note that

$$
\left\lfloor\frac{\left\lfloor\frac{n}{\beta_{1}}\right\rfloor}{\beta_{2}}\right\rfloor \leq\left\lfloor\frac{n}{\beta_{1} \beta_{2}}\right\rfloor,
$$

where $\beta_{1}, \beta_{2} \geq 1$ are real constants. Then the inequality (2.20) implies that

$$
\Gamma_{w}\left(\left\lfloor\frac{n}{\alpha^{j}}\right\rfloor\right) \leq 2 q^{2}\left(\frac{n}{\alpha^{j+1}}\right)^{3} \Gamma_{w}\left(\left\lfloor\frac{n}{\alpha^{j+1}}\right\rfloor\right) .
$$

From (2.20) and (2.21):

$$
\begin{aligned}
\Gamma_{w}(n) & \leq 2 q^{2}\left(\frac{n}{\alpha}\right)^{3} \Gamma_{w}\left(\left\lfloor\frac{n}{\alpha}\right\rfloor\right) \leq 2 q^{2}\left(\frac{n}{\alpha}\right)^{3} 2 q^{2}\left(\frac{n}{\alpha^{2}}\right)^{3} \Gamma_{w}\left(\left\lfloor\frac{n}{\alpha^{2}}\right\rfloor\right) \\
& \leq 2 q^{2}\left(\frac{n}{\alpha}\right)^{3} 2 q^{2}\left(\frac{n}{\alpha^{2}}\right)^{3} 2 q^{2}\left(\frac{n}{\alpha^{3}}\right)^{3} \Gamma_{w}\left(\left\lfloor\frac{n}{\alpha^{3}}\right\rfloor\right) \leq \ldots \\
& \leq\left(\prod_{j \geq 1}^{\left\lfloor\frac{\ln n}{\ln \alpha}\right\rfloor} 2 q^{2}\left(\frac{n}{\alpha^{j}}\right)^{3}\right) \Gamma_{w}(2) .
\end{aligned}
$$


Realize that

$$
\frac{n}{\alpha^{\left\lfloor\frac{\ln n}{\ln \alpha}\right\rfloor}} \geq 1 \text { and } \frac{n}{\alpha^{\left\lceil\frac{\ln n}{\ln \alpha}\right\rceil}} \leq 1
$$

Knowing that $\Gamma_{w}(2)=q$ and using Lemma 2.25 we obtain from $(2.22)$ :

$$
\Gamma_{w}(n) \leq\left(2 q^{2}\right)^{\frac{\ln n}{\ln \alpha}}\left(n^{\frac{\ln n}{2 \ln \alpha}}\right)^{3} \Gamma_{w}(2) \leq q\left(2 q^{2} n\right)^{\frac{3 \ln n}{2 \ln \alpha}}
$$

This ends the proof.

From Lemma 2.19 and Corollary 2.26 it follows easily:

Corollary 2.27. If $w \in R$ and $n>0$ then

$$
\left|\operatorname{Pal}_{w}(n)\right| \leq n q\left(2 q^{2} n\right)^{\delta \ln n}
$$

Remark 2.28. Although Corollary 2.26 requires $n>2$, it is easy to verify that Corollary 2.27 holds also for $n=\{1,2\}$. That is why we define $n>0$ in Corollary 2.27.

We can simply apply the upper bound for the palindromic complexity to construct an upper bound for the factor complexity:

Corollary 2.29. If $w \in R$ and $n>0$ then

$$
\left|\operatorname{Fac}_{w}(n)\right| \leq n^{4} q^{2}\left(2 q^{2} n\right)^{2 \delta \ln n}
$$

Proof. We apply again the property of rich words that every factor is determined by its longest palindromic prefix and its longest palindromic suffix [7]. If there are at most $t$ palindromic factors in $w$ of length $\leq n$, then clearly there can be at most $t^{2}$ different factors of length $n$. Let $\hat{\operatorname{Pal}}_{w}(k)=\max \left\{\left|\operatorname{Pal}_{w}(j)\right| \mid 0 \leq j \leq k\right\}$. From Corollary 2.27 we can deduce that

$$
t \leq \sum_{i=1}^{n}\left|\operatorname{Pal}_{w}(i)\right| \leq n \hat{\mathrm{Pal}_{w}}(n) \leq n^{2} q\left(2 q^{2} n\right)^{\log _{2} n}
$$

The corollary follows.

\section{RiCH WORDS CLOSED UNDER REVERSAL}

We can improve our upper bound for the factor complexity if we use the inequality (1.1). This inequality was shown for infinite words whose set of factors is closed under reversal. The next lemma and proposition generalize the existing proof for finite words $w \in A^{+}$with $\operatorname{Fac}_{w}(n+1)$ closed under reversal.

First we introduce an alphabet $B$ and an infinite word $\kappa(w)$. Let $B=A \cup\{x, y\}$ be an alphabet such that $x, y \notin A$; it follows that $|B|=|A|+2$. Given $w \in A^{+}$, let $\kappa(w)=\left(w x w^{R} y\right)^{\infty} \in B^{\infty}$.

We show that $\kappa(w)$ preserves richness.

Lemma 3.1. If $w \in A^{+}$is rich then $\kappa(w) \in B^{\infty}$ is also rich.

Proof. We have that $w x$ is rich, because $w$ is rich and $\operatorname{lps}(w x)=x$, which is a unioccurrent palindrome in $w x$ and $w x w^{R}$ is a palindromic closure of the rich word $w x$, which preserves richness [9]. As well $w x w^{R} y$ is rich, because $y$ is a unioccurrent palindrome in $w x w^{R} y$. Suppose that $\left(w x w^{R} y\right)^{j}$ is rich, where $j$ is a positive integer. We prove that $\left(w x w^{R} y\right)^{j+1}$ is rich. 
We have that $\operatorname{lps}\left(w x w^{R} y\right)^{j}=y\left(w x w^{R} y\right)^{j-1}$ and thus $\left(w x w^{R} y\right)^{j} w x w^{R}$ is a palindromic closure which is rich. Realize that $\operatorname{lps}\left(w x w^{R} y\right)^{j+1}=y\left(w x w^{R} y\right)^{j}$ and $y\left(w x w^{R} y\right)^{j}$ is unioccurrent in $y\left(w x w^{R} y\right)^{j+1}$. Thus $y\left(w x w^{R} y\right)^{j+1}$ is rich. It follows that all prefixes of $\kappa(w)$ are rich. Since all factors of rich words are rich, we proved that all factors of $\kappa(w)$ are rich. Consequently $\kappa(w)$ is rich. This completes the proof.

The following proposition generalizes the inequality (1.1) for finite words. It is known that for rich infinite words whose set of factors is closed under reversal, the inequality may be replaced with equality; this result has been proved in [6]. We prove also the equality for finite rich words.

Proposition 3.2. If $w \in A^{+}, \operatorname{Fac}_{w}(n+1)$ is closed under reversal, $|w| \geq n+1$, and $n>0$ then

$$
\left|\operatorname{Pal}_{w}(n)\right|+\left|\operatorname{Pal}_{w}(n+1)\right| \leq\left|\operatorname{Fac}_{w}(n+1)\right|-\left|\operatorname{Fac}_{w}(n)\right|+2 .
$$

If $w$ is also rich then the inequality becomes equality, formally:

$$
\left|\operatorname{Pal}_{w}(n)\right|+\left|\operatorname{Pal}_{w}(n+1)\right|=\left|\operatorname{Fac}_{w}(n+1)\right|-\left|\operatorname{Fac}_{w}(n)\right|+2 .
$$

Proof. Let $t=\kappa(w)$ and let $k \in\{n, n+1\}$. Clearly if $\operatorname{Fac}_{w}(n+1)$ is closed under reversal and $i \leq n$ that Fac $w(i)$ is also closed under reversal. Thus we have that

$$
\begin{aligned}
& \operatorname{Fac}_{t}(k)=\operatorname{Fac}_{w}(k) \cup\left\{u x v \mid u, v \in A^{*} \text { and } u \text { is a suffix of } w\right. \text { and } \\
& \left.v \text { is a prefix of } w^{R} \text { and }|u x v|=k\right\} \cup \\
& \left\{u y v \mid u, v \in A^{*} \text { and } u \text { is a suffix of } w^{R}\right. \text { and } \\
& v \text { is a prefix of } w \text { and }|u y v|=k\} \text {. }
\end{aligned}
$$

The formula (3.1) says that the set of factors of $t$ having length $k$ contains:

- the set of factors of $w$ of length $k$,

- the set of factors of $t$ containing one occurrence of $x$, and

- the set of factors of $t$ containing one occurrence of $y$.

It is easy to see that there are no other factors in $\operatorname{Fac}_{t}(k)$. Moreover for every $i \in\{0,1,2, \ldots, k-1\}$ there are unique $u \in \operatorname{Fac}_{w}(i)$ and $v \in \operatorname{Fac}_{w}(k-i-1)$ such that $u x v \in \operatorname{Fac}_{t}(k)\left(u y v \in \operatorname{Fac}_{t}(k)\right)$. It follows that

$$
\left|\operatorname{Fac}_{t}(k)\right|=\left|\operatorname{Fac}_{w}(k)\right|+2 k .
$$

Obviously $t$ contains exactly two palindromes $r_{1}, r_{2}$ such that $r_{1}, r_{2}$ are not factors of $w$ and $\left|r_{1}\right|=\left|r_{2}\right| \in$ $\{n, n+1\}$. In addition $r_{1}=u x u^{R}$ and $r_{2}=v y v^{R}$ for some words $u, v$. Formally

$$
\begin{array}{r}
\operatorname{Pal}_{t}(n+1) \cup \operatorname{Pal}_{t}(n)=\operatorname{Pal}_{w}(n+1) \cup \operatorname{Pal}_{w}(n) \cup\left\{u x u^{R}, v y v^{R} \mid u \text { is a suffix of } w\right. \text { and } \\
\left.v \text { is a suffix of } w^{R} \text { and }|u x u|=\left|v y v^{R}\right| \in\{n, n+1\}\right\}
\end{array}
$$

It follows that

$$
\left|\operatorname{Pal}_{t}(n+1)\right|+\left|\operatorname{Pal}_{t}(n)\right|=\left|\operatorname{Pal}_{w}(n+1)\right|+\left|\operatorname{Pal}_{w}(n)\right|+2 .
$$

Clearly Fac $_{t}$ is closed under reversal; realize that $t$ has infinitely many palindromic prefixes. Consequently (1.1) holds for $t$. Then from (1.1), (3.2), and (3.3) we have that

$$
\left|\operatorname{Pal}_{t}(n)\right|+\left|\operatorname{Pal}_{t}(n+1)\right| \leq\left|\operatorname{Fac}_{t}(n+1)\right|-\left|\operatorname{Fac}_{t}(n)\right|+2
$$


and

$$
\left|\operatorname{Pal}_{w}(n)\right|+\left|\operatorname{Pal}_{w}(n+1)\right|+2 \leq\left|\operatorname{Fac}_{w}(n+1)\right|+2(n+1)-\left|\operatorname{Fac}_{w}(n)\right|-2 n+2 .
$$

It follows from (3.4) that

$$
\left|\operatorname{Pal}_{w}(n)\right|+\left|\operatorname{Pal}_{w}(n+1)\right| \leq\left|\operatorname{Fac}_{w}(n+1)\right|-\left|\operatorname{Fac}_{w}(n)\right|+2 .
$$

If $w$ is rich then Lemma 3.1 implies that $t$ is rich. Then it follows from [6], (3.2), and (3.3) that

$$
\left|\operatorname{Pal}_{t}(n)\right|+\left|\operatorname{Pal}_{t}(n+1)\right|=\left|\operatorname{Fac}_{t}(n+1)\right|-\left|\operatorname{Fac}_{t}(n)\right|+2
$$

and

$$
\left|\operatorname{Pal}_{w}(n)\right|+\left|\operatorname{Pal}_{w}(n+1)\right|+2=\left|\operatorname{Fac}_{w}(n+1)\right|+2(n+1)-\left|\operatorname{Fac}_{w}(n)\right|-2 n+2 .
$$

It follows from (3.5) that

$$
\left|\operatorname{Pal}_{w}(n)\right|+\left|\operatorname{Pal}_{w}(n+1)\right|=\left|\operatorname{Fac}_{w}(n+1)\right|-\left|\operatorname{Fac}_{w}(n)\right|+2 .
$$

This completes the proof.

Based on Proposition 3.2 we can present a new relation for palindromic and factor complexity.

Proposition 3.3. Let $\hat{\operatorname{Pal}}_{w}(k)=\max \left\{\left|\operatorname{Pal}_{w}(j)\right| \mid 0 \leq j \leq k\right\}$. If $w \in R$ is a rich word such that $\operatorname{Fac}_{w}(n+1)$ is closed under reversal, $|w| \geq n+1$, and $n>0$, then

$$
\left|\operatorname{Fac}_{w}(n)\right| \leq 2(n-1) \hat{P a l}_{w}(n)-2(n-1)+q .
$$

Proof. Proposition 3.2 states for rich words that

$$
\left|\operatorname{Pal}_{w}(n)\right|+\left|\operatorname{Pal}_{w}(n+1)\right|-2=\left|\operatorname{Fac}_{w}(n+1)\right|-\left|\operatorname{Fac}_{w}(n)\right| .
$$

Since $\operatorname{Fac}_{w}(n+1)$ closed under reversal, we have that $\operatorname{Fac}_{w}(i)$ is closed under reversal for $i \leq n+1$. We can sum (3.6) over all lengths $i \leq n$ :

$$
\sum_{i=1}^{n-1}\left(\left|\operatorname{Pal}_{w}(i)\right|+\left|\operatorname{Pal}_{w}(i+1)\right|-2\right)=\sum_{i=1}^{n-1}\left(\left|\operatorname{Fac}_{w}(i+1)\right|-\left|\operatorname{Fac}_{w}(i)\right|\right) .
$$

The sums from (3.7) may be expressed as follows:

$$
\begin{aligned}
& \sum_{i=1}^{n-1}\left(\left|\operatorname{Fac}_{w}(i+1)\right|-\left|\operatorname{Fac}_{w}(i)\right|\right)=\operatorname{Fac}_{w}(2)-\operatorname{Fac}_{w}(1)+\operatorname{Fac}_{w}(3)-\operatorname{Fac}_{w}(2) \\
& \quad+\operatorname{Fac}_{w}(4)-\operatorname{Fac}_{w}(3)+\cdots+\operatorname{Fac}_{w}(n-1)-\operatorname{Fac}_{w}(n-2) \\
& \quad+\operatorname{Fac}_{w}(n)-\operatorname{Fac}_{w}(n-1)=\operatorname{Fac}_{w}(n)-\operatorname{Fac}_{w}(1) \\
& \\
& \sum_{i=1}^{n-1}\left(\left|\operatorname{Pal}_{w}(i)\right|+\left|\operatorname{Pal}_{w}(i+1)\right|-2\right) \leq(n-1)\left(\hat{\operatorname{Pal}_{w}}(n-1)+\hat{\operatorname{Pal}}_{w}(n)-2\right)
\end{aligned}
$$


From (3.7), (3.8), and (3.9) we get:

$$
\operatorname{Fac}_{w}(n)-\operatorname{Fac}_{w}(1) \leq(n-1)\left(\hat{\operatorname{Pal}_{w}}(n-1)+\hat{\operatorname{Pal}}_{w}(n)-2\right) .
$$

It follows that

$$
\operatorname{Fac}_{w}(n) \leq(n-1)\left(2 \hat{P a l}_{w}(n)-2\right)+\operatorname{Fac}_{w}(1) .
$$

This can be reformulated as:

$$
\operatorname{Fac}_{w}(n) \leq 2(n-1) \hat{\operatorname{Pal}}_{w}(n)-2(n-1)+\operatorname{Fac}_{w}(1) .
$$

Since $\operatorname{Fac}_{w}(1)=q$ it follows that

$$
\operatorname{Fac}_{w}(n) \leq 2(n-1) \hat{\mathrm{Pal}_{w}}(n)-2(n-1)+q .
$$

This completes the proof.

Proposition 3.3 and Lemma 2.27 imply an improvement to our upper bound for the factor complexity for rich words with $\operatorname{Fac}_{w}(n+1)$ closed under reversal:

Corollary 3.4. If $w \in R$ with $\operatorname{Fac}_{w}(n+1)$ closed under reversal, $|w| \geq n+1$, and $n>0$, then:

$$
\left|\operatorname{Fac}_{w}(n)\right| \leq 2(n-1) n q\left(2 q^{2} n\right)^{\delta \ln n}-2(n-1)+q .
$$

Since the palindromic closure of finite rich words is closed under reversal, we can improve the upper bound for factor complexity for finite rich words.

Corollary 3.5. If $w \in R$ and $n>0$ then

$$
\left|\operatorname{Fac}_{w}(n)\right| \leq 2(2 n-1) 2 n q\left(4 q^{2} n\right)^{\delta \ln 2 n}-2(2 n-1)+q
$$

Proof. Palindromic closure $\hat{w}$ of a word $w \in R$ preserves richness. Furthermore Fac $\hat{w}$ is closed under reversal, $\operatorname{Fac}_{w} \subseteq \operatorname{Fac}_{\hat{w}}$, and $|\tilde{w}| \leq 2|w|[9]$. Hence we can apply Corollary 3.4, where we replace $n$ with $2 n$.

Theorem 1.1 in the introduction presents a "simple" (although a somewhat worse) upper bound for the factor complexity. Here follows the proof.

Proof of Theorem 1.1. Note that for $n>0$ we have that

$$
2(2 n-1) 2 n q\left(4 q^{2} n\right)^{\delta \ln 2 n}-2(2 n-1)+q \leq 8 n^{2} q\left(4 q^{2} n\right)^{\delta \ln 2 n} \leq\left(4 q^{2} n\right)^{\delta \ln 2 n+2} .
$$

The theorem follows from Corollary 3.5.

Acknowledgements. The author wishes to thank to Štěpán Starosta for his useful comments. The author acknowledges support by the Czech Science Foundation grant GAČR 13-03538S and by the Grant Agency of the Czech Technical University in Prague, grant No. SGS14/205/OHK4/3T/14. 


\section{REFERENCES}

[1] J.-P. Allouche, M. Baake, J. Cassaigne and D. Damanik, Palindrome complexity. Theor. Comput. Sci. 292 (2003) 9-31.

[2] P. Baláži, Z. Masáková and E. Pelantová, Factor versus palindromic complexity of uniformly recurrent infinite words. Theor. Comput. Sci. 380 (2007) 266-275.

[3] L. Balková, Beta-integers and Quasicrystals, PhD thesis, Czech Technical University in Prague and Université Paris DiderotParis 7 (2008).

[4] L. Balková, E. Pelantová and Š. Starosta, Sturmian jungle (or garden?) on multiliteral alphabets. RAIRO: ITA 44 (2010) $443-470$.

[5] A. Blondin Massé, S. Brlek, S. Labbé and L. Vuillon, Palindromic complexity of codings of rotations. Theor. Comput. Sci. 412 (2011) 6455-6463.

[6] M. Bucci, A. De Luca, A. Glen and L.Q. Zamboni, A connection between palindromic and factor complexity using return words. Adv. Appl. Math. 42 (2009) 60-74.

[7] M. Bucci, A. De Luca, A. Glen and L.Q. Zamboni, A new characteristic property of rich words. Theor. Comput. Sci. 410 (2009) 2860-2863.

[8] X. Droubay, J. Justin and G. Pirillo, Episturmian words and some constructions of de Luca and Rauzy. Theor. Comput. Sci. 255 (2001) 539-553.

[9] A. Glen, J. Justin, S. Widmer and L.Q. Zamboni, Palindromic richness. Eur. J. Combin. 30 (2009) $510-531$.

[10] C. Guo, J. Shallit and A.M. Shur, Palindromic rich words and run-length encodings. Inform. Process. Lett. 116 (2016) $735-738$.

[11] D. Kosolobov, M. Rubinchik and A.M. Shur, $\mathrm{Pal}^{k}$ is linear recognizable online, in SOFSEM 2015: Theory and Practice of Computer Science, edited by G.F. Italiano, T. Margaria-Steffen, J. Pokorný, J.-J. Quisquater and R. Wattenhofer. Springer, Berlin Heidelberg (2015) 289-301.

[12] E. Pelantová and Š. Starosta, On words with the zero palindromic defect, in Combinatorics on Words, edited by S. Brlek, F. Dolce, C. Reutenauer and É. Vandomme. Springer International Publishing, Cham (2017) 59-71.

[13] E.A. Petrova and A.M. Shur, Transition property for cube-free words, in Computer Science - Theory and Applications, edited by R. van Bevern and G. Kucherov. Springer International Publishing, Cham (2019) 311-324.

[14] M. Rubinchik and A.M. Shur, The number of distinct subpalindromes in random words. Fund. Inform. 145 (2016) 371-384.

[15] J. Rukavicka, On the number of rich words, Developments in Language Theory: 21st International Conference, DLT 2017, Liège, Belgium, August 7-11, 2017. Proceedings, Springer International Publishing (2017) 345-352.

[16] J. Rukavicka, Transition property for $\alpha$-power free languages with $\alpha \geq 2$ and $k \geq 3$ letters, in Developments in Language Theory, edited by N. Jonoska and D. Savchuk. Springer International Publishing, Cham (2020) 294-303.

[17] L. Schaeffer and J. Shallit, Closed, palindromic, rich, privileged, trapezoidal, and balanced words in automatic sequences. Electr. J. Comb. 23 (2016) 1.25.

[18] J. Shallit and A. Shur, Subword complexity and power avoidance. Special issue in honor of the 70th birthday of Prof. Wojciech Rytter. Theor. Comp. Sci. 792 (2019) 96-116.

[19] J. Vesti, Extensions of rich words. Theor. Comput. Sci. 548 (2014) 14-24. 